\title{
Vittorio Fortunati, Sénac de Meilhan fra passato e futuro
}

Vittorio Fortunati e [paola perazzolo]

\section{(2) OpenEdition \\ 1 Journals}

\section{Edizione digitale}

URL: http://journals.openedition.org/studifrancesi/8273

DOI: 10.4000/studifrancesi.8273

ISSN: 2421-5856

Editore

Rosenberg \& Sellier

\section{Edizione cartacea}

Data di pubblicazione: 1 mai 2009

Paginazione: 179

ISSN: 0039-2944

\section{Notizia bibliografica digitale}

Vittorio Fortunati e [paola perazzolo], «Vittorio Fortunati, Sénac de Meilhan fra passato e futuro», Studi Francesi [Online], 157 (LIII | I) | 2009, online dal 30 novembre 2015, consultato il 09 janvier 2021. URL: http://journals.openedition.org/studifrancesi/8273 ; DOI: https://doi.org/10.4000/studifrancesi.8273

Questo documento è stato generato automaticamente il 9 janvier 2021.

\section{(c) (i) (9)}

Studi Francesi è distribuita con Licenza Creative Commons Attribuzione - Non commerciale - Non opere derivate 4.0 Internazionale. 


\title{
Vittorio Fortunati, Sénac de Meilhan fra passato e futuro
}

\author{
Vittorio Fortunati e [paola perazzolo]
}

\section{NOTIZIA}

VITTORIO FORTUNATI, Sénac de Meilhan fra passato e futuro, Pavia, ETS (Pubblicazioni della Facoltà di Lettere e Filosofia dell'Università di Pavia, n. 117) 2007, pp. 121.

1 Sénac de Meilhan (1736-1803) è un autore ancora poco studiato la cui fama è legata soprattutto a L'Emigré, romanzo epistolare del 1797 sugli esuli controrivoluzionari. In passato scarsamente riedito e abbastanza trascurato dalla critica, lo scrittore ha beneficiato del rinnovato interesse tributato alla letteratura del periodo in occasione del bicentenario della Rivoluzione. A prova di ciò, la recente pubblicazione di numerosi articoli e saggi e la riedizione di alcune opere, tra cui L'Emigré (a cura di M. Delon per "Folio classiques" nel 2004) e Les deux cousins (a cura di V. Fortunati per Champion nel 2007). Mancava tuttavia, per una migliore comprensione di quello che l'A. cita come uno «fra gli scrittori francesi più rappresentativi del periodo a cavallo fra XVIII e XIX secolo» (quarta di copertina), una monografia più completa e 'panoramica', che, pur concentrandosi su un aspetto preciso, prendesse in esame l'insieme dell'opera. Il presente lavoro colma questa lacuna, analizzando la produzione dello scrittore per riscontrarvi la coesistenza di vecchio e nuovo, mostrando come forme e strutture ormai usées veicolino in realtà idee innovative. Come si evince dal testo, nell'intera opera di Sénac la ripresa di generi e forme tradizionali o addirittura passate - il modello epistolare polifonico per L'Emigré, in cui Fortunati riscontra anche echi della narrativa eroica seicentesca, le autobiografie fittizie di gran moda alla fine del siècle d'or nel caso dei Mémoires d'Anne de Gonzague, Princesse Palatine, la struttura disomogenea e frammentaria tipica dei Caractères di La Bruyère per le Considérations sur l'esprit et les mours, il modello del conte orientale e philosophique di Les deux Cousins, novella per di più non esente da influenze fénéloniane - coesiste felicemente con l'espressione di temi e motivi che caratterizzeranno i primi decenni del secolo successivo - in particolare, 
una concezione "Sten-dhaliana" dell'amore, della società, dell'arte teatrale e un'interpretazione particolarmente lucida e attenta di cause, avvenimenti e prospettive della rivoluzione francese.

2 Stretto "fra passato e futuro", Sénac si rivela quindi un autore originale, un politologo acuto e intelligente nella disamina degli avvenimenti a lui coevi, una «figura intellettuale sostanzialmente coerente, le cui contraddizioni vengono a risolversi o si giustificano quale riflesso dei conflitti e delle ambiguità di un'epoca» (p. 10). Il lavoro di V. Fortunati ha quindi il merito non solo di contribuire a far meglio conoscere un autore ingiustamente poco studiato, ma anche di far luce su un periodo - quello tra il 1770 e il 1820 - le cui caratteristiche appaiono distintive e originali rispetto ai due secoli di cui assicura la transizione. Caratterizzato da una scrittura snella e piacevole, da una notevole precisione e ricchezza nell'analisi dei testi e nella scoperta di rimandi e influenze, il presente lavoro è di sicuro interesse ed importanza per la comprensione di un periodo anch'esso a lungo costretto "fra passato e futuro" - come si evince dalle due denominazioni più in uso, che giustamente Fortunati ritiene parziali: "svolta dei Lumi" e "preromanticismo" - ma ora finalmente oggetto di un interesse critico crescente. 